This item was submitted to Loughborough's Research Repository by the author.

Items in Figshare are protected by copyright, with all rights reserved, unless otherwise indicated.

\title{
Short sprints accumulated at school modulate postprandial metabolism in boys
}

PLEASE CITE THE PUBLISHED VERSION

PUBLISHER

Lippincott, Williams \& Wilkins

\section{VERSION}

AM (Accepted Manuscript)

\section{PUBLISHER STATEMENT}

This is a non-final version of an article published in final form in Smallcombe, J.W. ... et al., 2019. Short sprints accumulated at school modulate postprandial metabolism in boys. Medicine and Science in Sports and Exercise, 52(1):67-76, http://doi.org/10.1249/MSS.0000000000002121.

\section{LICENCE}

CC BY-NC-ND 4.0

\section{REPOSITORY RECORD}

Smallcombe, James, Laura Barrett, Lauren Sherar, Matthew J Sedgwick, Tommy Slater, and Keith Tolfrey. 2019. "Short Sprints Accumulated at School Modulate Postprandial Metabolism in Boys". figshare. https://hdl.handle.net/2134/9639302.v1. 


\title{
Medicine \& Science \\ IN \\ Sports \& Exercise
}

The Official Journal of the American College of Sports Medicine www.acsm-msse.org

... Published ahead of Print

\section{Short Sprints Accumulated at School Modulate Postprandial Metabolism in Boys}

\author{
James W Smallcombe ${ }^{1}$, Laura A Barrett ${ }^{1}$, Lauren B Sherar ${ }^{1}$, \\ Matthew J Sedgwick ${ }^{2}$, Tommy Slater ${ }^{1}$, Keith Tolfrey ${ }^{1}$ \\ ${ }^{1}$ School of Sport, Exercise and Health Sciences, Loughborough University, \\ Loughborough, United Kingdom; ${ }^{2}$ School of Social and Health Sciences, Leeds \\ Trinity University, Leeds, United Kingdom
}

Medicine \& Science in Sports \& Exercise ${ }_{\circledast}$ Published ahead of Print contains articles in unedited manuscript form that have been peer reviewed and accepted for publication. This manuscript will undergo copyediting, page composition, and review of the resulting proof before it is published in its final form. Please note that during the production process errors may be discovered that could affect the content. 


\title{
Short Sprints Accumulated at School Modulate Postprandial Metabolism in Boys
}

\author{
James W Smallcombe ${ }^{1}$, Laura A Barrett ${ }^{1}$, Lauren B Sherar ${ }^{1}$, Matthew J Sedgwick ${ }^{2}$, Tommy
}

$$
\text { Slater }^{1} \text {, Keith Tolfrey }{ }^{1}
$$

${ }^{1}$ School of Sport, Exercise and Health Sciences, Loughborough University, Loughborough, United Kingdom; ${ }^{2}$ School of Social and Health Sciences, Leeds Trinity University, Leeds, United Kingdom

Corresponding author: Dr Keith Tolfrey, School of Sport Exercise and Health Sciences, Loughborough University, Epinal Way, Loughborough, LE11 3TU. k.tolfrey@lboro.ac.uk, +44 (0)1509 226355

This research was supported by the National Institute for Health Research (NIHR) Leicester Biomedical Research Centre. The views expressed are those of the authors and not necessarily those of the NHS, the NIHR or the Department of Health. CONFLICT OF INTEREST: The authors declare no conflict of interest. The results of the present study do not constitute endorsement by ACSM. The results of the study are presented clearly, honestly, and without fabrication, falsification, or inappropriate data manipulation. 


\section{ABSTRACT}

Introduction: This study examined the efficacy of maximal sprint running accumulated during a typical school day to modulate postprandial metabolism in adolescent boys. Methods: Nineteen adolescent boys completed three, 2-day experimental conditions; a standard-practice control (CON); accumulated in-school sprint running (ACC); and a single block of afterschool sprint running (BLO). On Day 1, a fasting capillary blood sample was taken at 07:35 in the school. Three subsequent postprandial blood samples were taken at predetermined times after consumption of standardised breakfast and lunch. During ACC, participants accumulated four sets of $10 \times 30 \mathrm{~m}$ maximal-intensity sprint runs across natural breaks in lessons. During BLO, participants performed the same number of sprints (forty) in a single after-school exercise session. The blood samples from Day 1 were replicated on the day after exercise (Day 2). Results: On Day 1, no significant differences in total area under the plasma triacylglycerol concentration versus time curve (TAUC-TAG) were observed between conditions $(P=0.126)$. However, TAUC-insulin was lower in ACC compared with BLO $(-26 \%, \mathrm{ES}=0.86, P=0.001)$ and $\operatorname{CON}(-22 \%, \mathrm{ES}=0.72, P=0.010)$. On Day 2, TAUC-TAG was $12 \%$ lower after ACC $(\mathrm{ES}$ $=0.49 ; P=0.002)$ and $10 \%$ lower after BLO $(\mathrm{ES}=0.37 ; P=0.019)$ compared with CON. No significant differences were observed between conditions on Day 2 for postprandial insulin or glucose $(P \geq 0.738)$. Conclusion: Four sets of $10 \times 30 \mathrm{~m}$ sprints, accumulated in four separate bouts ( $<5 \mathrm{~min})$ during the school day reduced postprandial triacylglycerol and insulin concentrations in adolescent boys and may represent an effective in-school exercise strategy to promote metabolic health. Key words: adolescent; triacylglycerol; glucose; insulin; sprint running; accumulate 


\section{INTRODUCTION}

Regular exposure to elevated postprandial plasma triacylglycerol concentrations ([TAG]) has long been implicated in the development of atherosclerosis (1) and is now considered an independent risk factor for cardiovascular disease (2). Although atherosclerosis manifests typically in adulthood, atherogenesis is an insidious process known to be initiated much earlier, during childhood and adolescence $(3,4,5)$. Additionally, latest evidence points towards an alarming increase in prevalence of Type II diabetes in youth - the early onset of which is associated with premature development of complications and impaired life-quality (6). Consequently, it is important that interventions designed to preserve cardio-metabolic health are initiated during the early-stages of life. Encouragingly, compelling evidence indicates that an acute bout of moderate- to high-intensity exercise enhances postprandial lipaemic and glycaemic regulation in adolescents $(7,8)$. However, compliance to the current youth physical activity guidelines is poor in the UK, with only a small percentage of children meeting the recommended 60 minutes of moderate to vigorous exercise per day $(9,10)$. It is, therefore, crucial to identify modes of physical activity that young people find engaging and can incorporate realistically into their daily schedule. Given the substantial proportion of time spent at school, physical activity interventions that target this setting hold much promise. It is important that school-based physical activity programmes are optimised to promote both regular participation and maximise the cardio-metabolic benefit conferred. 
The notion of accumulating physical activity in short, manageable bouts throughout the day has gained considerable traction as an alternative means of achieving daily physical activity recommendations. The school-setting may be particularly amenable to physical activity accrual due to the regular, albeit short, breaks provided to children throughout the day. High-intensity interval exercise (HIIE), such as maximal sprint running, might be particularly suited to inschool participation due to the priority afforded to intensity over duration and, thus, its purported time-efficiency (11). Furthermore, this form of physical activity is associated with lower levels of fatigue in paediatric populations compared with adults (12) and may resemble more closely the spontaneous, intermittent nature of habitual physical activity patterns of children and adolescents $(13,14)$. It is also possible that maximal sprint exercise is less susceptible to the monotony often experienced by young people when performing moderate-intensity continuous exercise $(15,16)$ - an issue that is particularly pertinent when the potential for long-term exercise adherence is considered. However, perhaps the most compelling rationale for high-intensity sprint exercise is that it may provide a viable alternative to more "traditional" forms of exercise (e.g. athletics, team sports etc.) and promote more varied physical activity engagement during childhood and adolescence (17). Encouragingly, high intensity sprinting has been successfully employed as an exercise tool in a range of settings with both healthy and "at risk" paediatric populations (18), including those presenting with overweight and obesity, suggesting that it may be suitable for use across a wide spectrum of children and adolescents.

A growing body of paediatric exercise research supports the efficacy of HIIE to induce desirable postprandial metabolic responses. Reduced postprandial [TAG] and enhanced endothelial function were observed 14 hours after a single session of maximal sprint cycling in adolescent 
boys (19). Reduced postprandial [TAG] was also reported in adolescent boys (20) and girls (21) the day after a bout of low-volume high-intensity interval running. Improved glycaemic regulation was also observed the day of HIIE performance. Glucose tolerance and insulin sensitivity were both improved in adolescent boys during an oral glucose tolerance test (OGTT) administered immediately after high intensity cycling $(8 \times 1$ min cycling bouts at $90 \%$ of peak power) (8). In addition, in a separate study using a similar experimental protocol, Bond and colleagues (22) observed lower postprandial glucose concentrations when sprint cycling was accumulated in four separate bouts across the day. However, beyond these limited findings, research that has explored the effect of accumulated HIIE on postprandial metabolism during youth is sparse and has, to date, failed to bridge the gap between the laboratory and "the field". It is not known currently if it is feasible to incorporate HIIE into a typical school day and whether such in-school interventions can promote metabolic health during childhood and adolescence. The current appetite for school-based exercise programmes is exemplified by the success of the Daily Mile running initiative (23); an intervention model which may serve as a template for the incorporation of alternative forms of exercise (e.g. shorter, higher intensity running bouts) into the school day.

To the authors' knowledge, no previous study has examined the efficacy of accumulated HIIE using a two-day experimental model; whereby the effect of high-intensity interval exercise is examined 12-24 hours after the performance of exercise. This time window is particularly important as it aligns with the exercise-induced upregulation of lipoprotein lipase activity and, thus, a period in which enhanced clearance of circulating [TAG] is most likely to be observed (24). Furthermore, no previous study has compared the effects HIIE accumulated in short bouts 
across the school day with those induced by the same dose of exercise performed during an uninterrupted exercise session. Furthermore, it is also unknown if the metabolic response from HIIE accumulated during the day is comparable to the response exhibited when the same volume and intensity of exercise is performed during a single bout of exercise.

In light of the aforementioned limitations of the previous research, the present school-based study aimed to: 1) examine the effect of maximal-intensity sprint running accumulated in multiple bouts during a typical school day (ACC) on postprandial metabolism; 2) compare the effects of accumulated maximal-intensity sprint running - both on the day of exercise and on the day after - with those of the same dose of exercise performed in a single, after-school exercise session (BLO).

\section{METHODS}

After institutional ethical approval, 19 healthy adolescent boys volunteered for the study and completed all measures (i.e., only 19 volunteers and no drop-outs). These participants were recruited from a local secondary school after their attendance at a school-based presentation. Prospective participants were eligible for study inclusion providing they were: 11 to 14 years old; were not taking prescribed medication that might moderate postprandial metabolism; did not have a pre-existing injury or medical condition which precluded very hard exercise; and did not have a nut or dairy food allergy. Written assent was obtained from each participant and written informed consent was obtained from a parent or guardian. Suitability for admittance into the 
study was confirmed by the completion of a general health screen questionnaire. Participant characteristics are presented in Table 1.

\section{Anthropometry and preliminary exercise measurements}

Anthropometry was conducted with participants wearing shorts, T-shirt and socks. Body mass was measured to the nearest $0.1 \mathrm{~kg}$ using a digital scale and stature was measured to the nearest $0.01 \mathrm{~m}$ using a wall-mounted stadiometer (Holtain, Crosswell, UK). Triceps and subscapular skinfold thicknesses were measured on the right-hand side of the body to the nearest $0.2 \mathrm{~mm}$ using Harpenden callipers (John Bull, St. Albans, UK). The skinfold thickness was calculated as the median of three measurements. Percentage body fat $(\% \mathrm{BF})$ was estimated using maturation, race and sex-specific equations (25). Waist circumference was measured midway between the $10^{\text {th }}$ rib and the iliac crest (26). Physical maturity was estimated through a five-point selfassessment of secondary sexual characteristics (27). Scientific photographs depicting the five stages of genital and pubic hair development, ranging from 1 indicating pre-pubescence to 5 indicating full sexual maturity, were used privately by the participants to provide this information.

\section{Preliminary exercise measures}

Before the preliminary exercise tests, participants were familiarised with exercising on the treadmill ergometer (Mercury Medical, h/p/cosmos sports \& medical Gmbh, Germany). Shortrange telemetry (PE4000, Polar-Electro, Kempele, Finland) was used to monitor HR continuously throughout the exercise tests. Peak heart rate $\left(\mathrm{HR}_{\text {peak }}\right)$ was defined as the highest 
HR recorded during the test. Ratings of perceived exertion (RPE) were measured during the final 15 s of each exercise stage using the pictorial OMNI (0 to 10) scale (28).

Peak $\dot{\mathrm{V}}_{2}$ was determined using an incremental gradient-based treadmill protocol. Each participant ran at comfortable fixed speed $\left(8.0\right.$ to $\left.10.5 \mathrm{~km} \cdot \mathrm{h}^{-1}\right)$ as selected by the investigators based on performance during a familiarisation treadmill run. Expired air was collected into Douglas bags during each successive minute of exercise via open-circuit spirometry. The treadmill belt gradient was raised by $1 \%$ every minute until volitional exhaustion was attained. Due to the limited number of children (20-40\%) that display a plateau in their $\dot{\mathrm{V}} \mathrm{O}_{2}$ when performing exercise to exhaustion, and to avoid the possible acceptance of a 'submaximal peak $\dot{\mathrm{V}}_{2}{ }^{\prime}$ based on secondary criteria (29), after a ten-minute recovery period each participant completed an additional verification stage to volitional exhaustion (30), performed at $\sim 110 \%$ of the work rate achieved during the initial incremental exercise test. Typically, participants completed 2 to 3 minutes of running during the verification phase, and of the 19 participants in the sample, only four attained an oxygen consumption higher than that achieved during the initial incremental exercise test. In such cases, oxygen consumption attained during the verification phase was accepted as peak $\dot{\mathrm{V}} \mathrm{O}_{2}$.

A paramagnetic oxygen $\left(\mathrm{O}_{2}\right)$ analyser and infrared carbon dioxide $\left(\mathrm{CO}_{2}\right)$ analyser (Servomex, Sussex, UK) were used to determine the concentration of $\mathrm{O}_{2}$ and $\mathrm{CO}_{2}$ in the expired air samples. The volumes of expired gas were determined using a dry gas meter (Harvard Apparatus, Kent, UK) and were corrected to standard temperature and pressure (dry). For each expired gas sample, 
oxygen uptake $\left(\dot{\mathrm{V}}_{2}\right)$, expired carbon dioxide $\left(\dot{\mathrm{V} C O}_{2}\right)$, minute ventilation $\left(\dot{\mathrm{V}}_{\mathrm{E}}\right)$, and respiratory exchange ratio were calculated.

\section{Experimental design}

A within-measures, counter-balanced research design was employed whereby all participants completed three, 2-day experimental conditions; a standard-practice control condition (CON); an accumulated, maximal sprint running exercise condition performed across the school day (ACC); and a single block, maximal sprint running exercise condition performed after-school (BLO). Standardised washout periods of 14 days separated the three experimental conditions. Participants completed each experimental condition in small groups (four groups of 4 participants, and one group of 3 participants) with counterbalancing achieved at the group level. To enable the simultaneous drawing of blood samples from multiple participants at standardised time points, a team of four researchers - all fully trained in the blood sampling technique - was assembled. The experimental study design is presented schematically in Figure 1a \& 1 b.

\section{Day 1 (intervention day)}

After a standardised overnight fast, participants arrived at the school at 07:20. A fasting capillary blood sample was taken at 07:35. Three subsequent blood samples were taken in the postprandial state at 10:30, 12:35 and 15:10 (these were during natural breaks and at the end of the school day). In all conditions, a standardised test-breakfast and test-lunch were consumed at 08:10 and 13:00. 
During CON, no exercise was prescribed on Day 1; participants attended school as normal and only presented for blood samples as described above. During ACC, participants accumulated four sets of $10 \times 30 \mathrm{~m}$ maximal-intensity sprint runs, interspersed by $15 \mathrm{~m}$ active recovery walks, across natural breaks in lessons on Day 1 (07:50, 10:35, 12:40 and 15:30; 40 sprints in total). During BLO, participants also completed 40 sprints but in a single block of exercise starting at 16:00 on Day 1. The exercise during BLO was completed in four sets of $10 \times 30 \mathrm{~m}$ sprints (interspersed by $15 \mathrm{~m}$ active recovery walks) with 5, 6 and $7 \mathrm{~min}$ of passive recovery between sets, respectively. The participants completed the sprints, both ACC and BLO, in standard school uniform with training shoes (sneakers), removing only the blazer for improved comfort. To avoid large individual participant discrepancies in sprint performance during ACC and BLO, participants were grouped based on individual sprint capabilities, as guided by the recommendation of the participant's Physical Education teacher.

During all bouts of exercise, heart rate was monitored continuously (PE4000, Polar-Electro, Kempele, Finland), and participants were asked to rate their affective perception using the OneItem Feeling Scale (-5 to +5) (31), following each set of sprints in ACC and BLO.

\section{Day 2 (Post-intervention)}

After a standardised overnight fast, participants arrived at school at 07:30. A fasting capillary blood sample was taken at 07:55. Three subsequent blood samples were taken in the postprandial state at 10:35, 13:10, and 15:10. A high fat test-breakfast and test-lunch, both standardised to body mass, were consumed at $08: 10$ and 12:40, respectively. For the duration of the day, 
participants adhered to their normal school timetable. Day 2 is presented schematically in Figure $1 b$.

\section{Standardisation of diet and physical activity}

To ensure each participant commenced the three experimental conditions in a similar metabolic state, dietary intake and physical activity were standardised during the 24-hours preceding Day 1 (Day 0) of the first condition. Participants were asked to complete a parent-aided weighed food diary documenting free-living dietary intake and minimise their level of physical activity (i.e. refrain from structured exercise and active participation in physical education (PE) lessons). Participants were asked to replicate this behaviour on Day 0 of the subsequent exercise trials; this was confirmed verbally. Participants completed weighed food diaries using digital kitchen scales (Andrew James UK Ltd., Bowburn, UK), and the CompEat Pro 5.8.0 computerized food tables (Nutrition Systems, London, UK) were used to analyse dietary intake subsequently. Physical activity was quantified via accelerometry (ActiGraph GT1M; ActiGraph, Pensacola, Florida). The accelerometer was worn on the right hip during waking hours (removed for waterbased activities). Raw ActiGraph data files were analyzed using custom-made data reduction software (KineSoft Software, version 3.3.76, Loughborough University, UK; http://www.kinesoft.org). During data processing, 5-s epoch data were reintegrated to 60-s epochs; 60 min of consecutive zeros, allowing for $2 \mathrm{~min}$ of nonzero interruptions, was used to remove non-wear, and a minimum of 8 -h of valid wear time was required for a valid day. Physical activity was interpreted using age-specific intensity cut points. 
A cereal bar (10 g carbohydrate, $1 \mathrm{~g}$ fat, $1 \mathrm{~g}$ protein and $272 \mathrm{~kJ})$ was consumed before 21:00 on Day 0 to standardise the overnight fasting period. On Day 1 of each exercise condition, a standardised evening meal was provided to participants and was consumed between 17:00 and 21:00 that evening. This mixed evening meal consisted of pasta, tomato-based sauce, cheese, orange juice and a chocolate biscuit (25.8 g fat, $137.2 \mathrm{~g}$ carbohydrate, $31.5 \mathrm{~g}$ protein and 3792 $\mathrm{kJ})$. If participants were unable to eat all of the food provided for the evening meal on Day 1 of the first experimental condition, they were asked to weigh and record the leftovers to enable precise replication on Day 1 of all subsequent conditions. A cereal bar was consumed before 21:00 on Day 1 to standardise the overnight fasting period. Following consumption of the cereal bar, participants drank only water, until the test-breakfast, which was provided on the morning of Day 2. On both Day 1 and Day 2 (the experimental window) of each experimental condition, participants were asked to minimise incidental physical activity and refrain from participation in Physical Education lessons and extracurricular sports (in order to minimise the confounding effect of variable physical activity on the postprandial blood measures).

\section{Test meals}

On Day 1 of all experimental conditions, participants were provided with a standardised, mixed macronutrient breakfast and lunch, designed to reflect typical meal consumption at school. The breakfast consisted of cereal, milk and fruit juice $(3.3 \mathrm{~g}$ fat, $80.0 \mathrm{~g}$ carbohydrate, $8.3 \mathrm{~g}$ protein and $1603 \mathrm{~kJ}$ ). The lunch consisted of white bread, butter, chicken, potato crisps, chocolate biscuits and fruit juice (45.5 g fat, $129.7 \mathrm{~g}$ carbohydrate, $35.1 \mathrm{~g}$ protein and $4474 \mathrm{~kJ})$. An apple was provided as an after-school snack ( $15.7 \mathrm{~g}$ carbohydrate, $0.1 \mathrm{~g}$ fat, $0.5 \mathrm{~g}$ protein and $276 \mathrm{~kJ})$. 
On Day 2 of all conditions, participants were given a high-fat test breakfast and lunch prescribed relative to body mass. The test breakfast consisted of croissants, chocolate spread, whole milk, double cream and milkshake powder (1.6 g fat (60\% of total energy), $1.8 \mathrm{~g}$ carbohydrate (33\%), $0.4 \mathrm{~g}$ protein (7\%) and $95 \mathrm{~kJ}$ energy per kilogram body mass). The test lunch consisted of white bread, mild cheddar cheese, butter, potato crisps, whole milk and milkshake powder (1.1 g fat (50\%), 1.9 g carbohydrate (38\%), 0.6 g protein (12\%) and $86 \mathrm{~kJ}$ energy per kilogram of body mass).

\section{Analytical methods}

Before the collection of capillary blood samples, the whole hand was submerged in $40^{\circ} \mathrm{C}$ water for $5 \mathrm{~min}$ and then dried thoroughly before the fingertip was pierced (Unistick 3 Extra, Owen Mumford, UK). The first drop of blood was discarded before 300 to $600 \mu \mathrm{L}$ of blood was collected in potassium-EDTA-coated microvette tubes (Sarstedt Ltd., Leicester, UK). The whole blood was centrifuged immediately at 12,800 g for 15 min (Eppendorf 5415c, Hamburg, Germany). The resulting plasma sample was stored at $-20^{\circ} \mathrm{C}$ for subsequent analysis. Plasma [TAG] and [glucose] were determined by a benchtop analyser (Pentra 400; HORIBA ABX Diagnostics, Montpellier, France) using enzymatic, colorimetric methods (HORIBA ABX Diagnostics, Montpellier, France). The within-batch coefficients of variation for [TAG] and [glucose] were $1.4 \%$ and $0.5 \%$, respectively. Plasma insulin concentrations were determined using a commercially available enzyme-linked immunosorbent assay (Mercodia Insulin ELISA 
10-1113-01, Mercodia AB, Uppsala, Sweden). The within-batch coefficient of variation for plasma insulin concentration analysis was $4.2 \%$.

Acute changes in plasma volume were estimated from haemoglobin concentration and haematocrit ascertained from the fasting and final blood samples. Haemoglobin concentration was determined via the cyanmethemoglobin method; $20 \mu \mathrm{L}$ of whole blood was added to $5 \mathrm{~mL}$ of Drabkin's reagent and the absorbance was quantified via photometry at a wavelength of 546 nm (Cecil CE1011; Cecil instruments, Cambridge, UK). A microhaematocrit centrifuge and reader (Haematospin 1300 Microcentrifuge; Hawksley and Sons Ltd., Sussex, UK) were used to quantify haematocrit.

\section{Sample size calculation}

Based on published data from our laboratory (32), we calculated a priori that a sample size of 19 participants was required to complete our three condition within measures study to detect a main effect of condition for Day 2 time-averaged TAUC-TAG. This approach assumed $80 \%$ power, a $20 \%$ difference $(0.27 \mathrm{mmol} / \mathrm{L}$ per $\mathrm{h})$ between the control and either exercise condition, with a standard deviation of 0.53 (thus standardised difference of 0.51 ), an intra-person correlation coefficient of 0.70 and an alpha error rate of $5 \%$. 


\section{Statistical analyses}

The Statistical Package for Social Sciences (SPSS) software version 23.0 for Windows (SPSS Inc., Chicago, IL, USA) was used for all data analyses. The trapezium rule was used to calculate total area under the plasma concentration versus time curve for TAG (TAUC-TAG), glucose (TAUC-glucose) and insulin (TAUC-insulin) for all experimental conditions. Homeostatic Model Assessment of Insulin Resistance (HOMA-IR) was determined on Day 1 and Day 2 from fasting plasma glucose and insulin concentrations based on the calculations outlined by Matthews et al. (33). Normality of the data was checked using Shapiro Wilk tests. Normally distributed data are presented as mean (SD). Data for free-living physical activity and sedentary time, and concentrations of plasma TAG, glucose and insulin were natural log transformed before analyses. These data are presented as geometric mean (95\% confidence interval) and analyses are based on ratios of geometric means and $95 \%$ confidence intervals (CI) for ratios. Linear mixed models repeated for condition were used to examine differences in dietary intake, free living physical activity and sedentary time (wear time included as a covariate), plasma volume changes, fasting concentrations, HOMA-IR and TAUC responses. Differences in postprandial [TAG], [glucose] and [insulin] and exercise responses to ACC and BLO were examined using linear mixed models repeated for condition and time. Where appropriate, to supplement key findings, absolute standardised effect sizes (ES) were calculated for withinmeasures comparisons as follows:

$$
\mathrm{ES}=\frac{\text { mean } v 2-\text { mean } v 1}{\mathrm{CONSD}}
$$


Where $v 1$ and $v 2$ represent the two variable mean values being compared and the CON SD is the standard-practice control condition standard deviation. In the absence of a clinical anchor, an ES of 0.2 was considered to be the minimum important difference, 0.5 moderate and 0.8 large (34).

\section{RESULTS}

\section{Dietary intake}

Average free-living energy intake did not differ significantly on Day 0 of ACC, BLO and CON

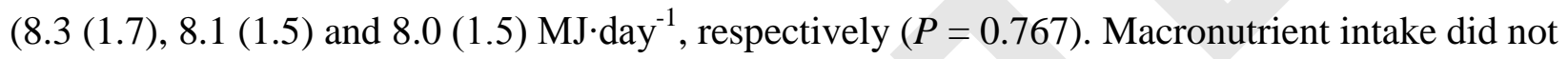
differ significantly between ACC, BLO and CON for carbohydrate (289 (44), 276 (47) and 287

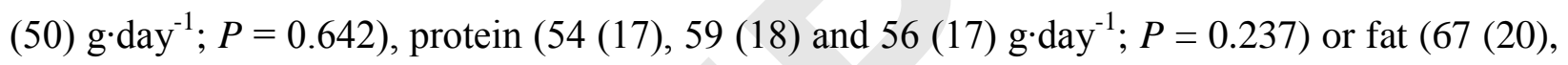
66 (23) and $\left.61(24) \mathrm{g} \cdot \mathrm{day}^{-1} ; P=0.301\right)$, respectively.

\section{Free-living physical activity and sedentary time}

Average accelerometer wear time did not differ significantly between conditions $(\mathrm{ACC}=11.9 \mathrm{~h}$; $\mathrm{BLO}=12.5 \mathrm{~h} ; \mathrm{CON}=11.7 \mathrm{~h} ; P=0.426)$. No significant differences were observed for freeliving accelerometer counts per minute $(P=0.792)$, sedentary time $(P=0.696)$, light $(P=$ $0.175)$, moderate $(P=0.824)$ or vigorous intensity physical activity $(P=0.727)$ on Day 0 of ACC, BLO and CON.

\section{Exercise responses to $A C C$ and $B L O$}

All participants completed all sprint repetitions successfully during both ACC and BLO exercise conditions. Each $30 \mathrm{~m}$ sprint repetition was completed in approximately 6 seconds, with each set 
of 10 sprints completed within approximately 5 minutes, including time spent in recovery (ACC: Set $1=5: 07$, Set $2=4: 52$, Set $3=4.50$, Set $4=5: 12$; BLO: Set $1=4: 58$, Set $2=4: 32$, Set $3=$ $4: 36$, Set $4=4: 38$ ). Average heart rate and affective responses to maximal sprint running across sets during ACC and BLO are presented in Table 2. Average heart rate did not differ significantly within sets of sprints $(P=0.087)$ but was $4 \%$ higher during BLO (182 (7) beats $\left.\cdot \min ^{-1}\right)$ compared with ACC $\left(176(8)\right.$ beats $\left.\cdot \min ^{-1}\right)(95 \% \mathrm{CI}=3$ to $5 \%, P<0.001$, ES = 0.68). The condition by set interaction was not significant, showing that subtle variations in mean heart rate across the four sets were similar in ACC and BLO $(P=0.996)$. The condition by sprint set interaction was significant $(P=0.002)$ for affective ratings on the One Item Feeling Scale; there was a between condition divergence in the last two sets with ratings deteriorating in BLO compared with ACC.

\section{Plasma volume changes}

On Day 1 and Day 2, changes in plasma volume between fasting and final blood samples were small and did not vary significantly between the three experimental conditions $(\leq 1.6 \%, P \geq$ 0.717). Therefore, further statistical analyses were completed without adjustment to the raw plasma [TAG], [glucose] and [insulin].

\section{Fasting [TAG], [insulin], [glucose] and HOMA-IR}

No significant differences were observed between conditions for fasting $[\mathrm{TAG}]$ on Day $1(P=$ 0.134) ACC $0.64 \mathrm{mmol} \cdot \mathrm{L}^{-1}$; BLO $0.69 \mathrm{mmol} \cdot \mathrm{L}^{-1}$; $\mathrm{CON} 0.61 \mathrm{mmol} \cdot \mathrm{L}^{-1}$ or Day $2(P=0.187)$ ACC $0.67 \mathrm{mmol} \cdot \mathrm{L}^{-1}$; $\mathrm{BLO} 0.65 \mathrm{mmol} \cdot \mathrm{L}^{-1}$; $\mathrm{CON} 0.71 \mathrm{mmol} \cdot \mathrm{L}^{-1}$. 
No significant differences were observed between conditions for fasting [insulin] on Day $1(P=$ $0.905) \mathrm{ACC}$ vs $\mathrm{BLO}$ vs $\mathrm{CON}=48.6$ vs 50.0 vs $47.7 \mathrm{pmol} \cdot \mathrm{L}^{-1}$, respectively or Day $2(P=0.468)$ ACC vs $\mathrm{BL}$ vs $\mathrm{CON}=43.5$ vs 42.4 vs $46.5 \mathrm{pmol} \cdot \mathrm{L}^{-1}$, respectively. Similarly, no significant differences were observed between conditions for HOMA-IR on Day $1(P=0.874)$ ACC vs BLO vs CON $=1.74$ vs 1.83 vs 1.76 , respectively or Day $2(P=0.238)$ ACC vs BLO vs CON $=$ 1.68 vs 1.61 vs 1.85 , respectively.

No significant differences were observed for fasting [glucose] on Day $1(P=0.411)$ ACC 5.03 $\mathrm{mmol} \cdot \mathrm{L}^{-1}$; BLO $4.95 \mathrm{mmol} \cdot \mathrm{L}^{-1}$; $\mathrm{CON} 4.97 \mathrm{mmol} \cdot \mathrm{L}^{-1}$. However, a significant difference was observed on Day $2(P=0.007)$ ACC $5.26 \mathrm{mmol} \cdot \mathrm{L}^{-1}$; $\mathrm{BLO} 5.14 \mathrm{mmol} \cdot \mathrm{L}^{-1}$; CON $5.38 \mathrm{mmol} \cdot \mathrm{L}^{-1}$; based on ratios of geometric means, fasting [glucose] was $4 \%$ lower after BLO (95\% CI $=-7$ to $2 \%, \mathrm{ES}=0.85, P=0.002)$ compared with CON. The differences between ACC and CON $(\mathrm{ES}=$ $0.43, P=0.113)$ and $\mathrm{BLO}$ and $\mathrm{ACC}(\mathrm{ES}=0.43, P=0.105)$ were smaller and non-significant.

\section{Plasma [TAG], [glucose] and [insulin] in the postprandial period}

Plasma [TAG] responses across time and between conditions are presented in Figure 2. On Day 1, the main effect for condition $(P=0.126)$ and condition by time interaction $(P=0.850)$ were not significant. In contrast, significant main effects for condition were observed on Day 2 with [TAG] $9 \%$ lower after both ACC $(95 \% \mathrm{CI}=-15$ to $-3 . \mathrm{ES}=0.34, P=0.004)$ and $\mathrm{BLO}(95 \% \mathrm{CI}$ $=-15$ to $-3, \mathrm{ES}=0.34, P=0.003)$ compared with CON. The difference between BLO and ACC was trivial $(95 \% \mathrm{CI}=-7$ to $6, \mathrm{ES}=0.01, P=0.913)$. 
TAUC-TAG for all conditions on both days are presented in Table 3. The differences between conditions on Day 1 were not significant $(P=0.359)$. However, on Day 2 TAUC-TAG was $12 \%$ lower after $\mathrm{ACC}(95 \% \mathrm{CI}=-19$ to $-5, \mathrm{ES}=0.49 ; P=0.002)$ and $10 \%$ lower after $\mathrm{BLO}(95 \% \mathrm{CI}$ $=-17$ to $-2, \mathrm{ES}=0.37 ; P=0.019)$ compared with $\mathrm{CON}$. The difference between $\mathrm{BLO}$ and ACC was trivial $(95 \% \mathrm{CI}=-5$ to $12, \mathrm{ES}=0.12, P=0.418)$.

Plasma [insulin] responses across time and between conditions are presented in Figure 2. On Day 1, the main effect for condition was significant $(P=0.003)$; pairwise effects showed ACC was $15 \%$ lower than $\mathrm{CON}(95 \% \mathrm{CI}=-25$ to $-4, \mathrm{ES}=0.43, P=0.010)$ and $19 \%$ lower than BLO $(95 \% \mathrm{CI}=-28$ to $-8, \mathrm{ES}=0.55, P=0.001)$. However, the difference between BLO and CON was trivial $(95 \% \mathrm{CI}=-8$ to $18, \mathrm{ES}=0.11, P=0.507)$ and the condition by time interaction was not significant $(P=0.474)$. Similarly, the main effect for condition $(P=0.780)$ and condition by time interaction $(P=0.906)$ were not significant on Day 2 .

TAUC-insulin for all conditions and on both days is presented in Table 3. On Day 1, TAUCinsulin was $22 \%$ lower during ACC compared with $\mathrm{CON}(95 \% \mathrm{CI}=-32$ to $-10, \mathrm{ES}=0.53, P=$ 0.001). Similarly, on Day 1 TAUC-insulin was $26 \%$ lower during ACC compared with BLO (95\% $\mathrm{CI}=-36$ to $-14, \mathrm{ES}=0.63 P \leq 0.001)$. The difference between $\mathrm{BLO}$ and $\mathrm{CON}$ was trivial $(95 \% \mathrm{CI}=-9$ to $21, \mathrm{ES}=0.10, P=0.490)$. There were no significant between condition differences in TAUC-insulin on Day $2(P=0.771)$. 
Plasma [glucose] responses across time and between conditions are presented in Figure 2. No significant differences were observed for postprandial [glucose] across conditions on Day 1 or Day 2 (main effect condition, $P \geq 0.944$; condition by time interaction, $P \geq 0.844$ ). Similarly, no differences in TAUC-glucose (Table 3) were observed across conditions on Day 1 or Day 2 ( $P \geq$ $0.738)$.

\section{DISCUSSION}

The primary finding of the current study was that $40 \times 30 \mathrm{~m}$ sprints, accumulated in four separate bouts $(\approx 4.5$ min per set) during the school day reduced next day postprandial plasma [TAG] in apparently healthy, adolescent boys. Furthermore, the reductions in postprandial lipaemia observed after accumulated exercise were similar in magnitude to those observed after the same dose of exercise was performed in a single after-school exercise block $(\approx 36$ min duration $)$. In addition, postprandial insulin concentrations were reduced on the day of accumulated sprint performance. This is the first study to examine the acute effects of accumulated sprint running incorporated into a typical school day. The findings demonstrate the potential for high-intensity exercise accumulated at school to promote metabolic health during adolescence.

The small-to-moderate exercise-induced reductions in TAUC-TAG observed in school after both ACC and BLO were very similar to those reported after HIIE previously. Thackray and colleagues $(20,35)$ reported a moderate $(E S=0.50) 11 \%$ reduction in TAUC-TAG after highintensity treadmill interval running in 11- to 12-year-old boys and a small $(\mathrm{ES}=0.30) 10 \%$ reduction in 12- to 13-year-old girls, respectively. Similarly, a small $(\mathrm{ES}=0.40) 13 \%$ reduction 
was reported in adolescent boys following maximal effort cycling intervals performed during a 90-minute exercise session (19). In contrast, the repeated $30 \mathrm{~m}$ sprint running bouts performed in the current study were extremely short with each sprint effort completed in $<6$ seconds and each set of sprints completed in approximately 4.5 minutes, including recovery between sprint bouts. Furthermore, the exercise was performed between school lessons without disruption to the school day. This type of short-duration sprint running may better reflect the intermittent activity patterns thought to be preferred by children and adolescents (15) and may, therefore, be more conducive to long-term exercise adherence than HIIE protocols involving longer exercise bouts (e.g. $\geq 1 \mathrm{~min})(20,35,22,8)$. When the current findings are considered alongside those from previous studies, the reductions in [TAG] observed after HIIE or sprint cycling are consistent, and similar to the small to moderate reductions reported after continuous moderate-intensity exercise (7). Energy expenditure has been proposed as an important determinant of the exerciseinduced reduction in postprandial TAG for adults (36), although its relative contribution has been questioned (37). Although the nature of HIIE - particularly when performed "in the field" precludes direct quantification of energy expenditure, it is likely that the energy expended during the very short-duration sprints was fairly modest compared to that expended during prolonged moderate-intensity continuous exercise protocols (i.e., 1 to $2.5 \mathrm{MJ}$ ) (7). Therefore, the data emerging from HIIE studies, in which total energy expenditure is likely modest, suggest that exercise-intensity per se may also be an important mediator of the exercise-induced reduction in [TAG].

In agreement with findings derived from previous one-day experimental models $(22,8)$, no differences in circulating [TAG] were observed on the day of sprint performance (Day 1). This is 
likely due to the time-course of lipoprotein lipase (LPL) activity which appears to peak 12 to 18 hours after exercise (38) and is thought to play an important role in increasing the clearance of circulating TAG (24). Therefore, the use of a two-day experimental model, which captures the time frame of this mechanism, represents a major strength of the current study.

No between condition differences in postprandial glucose concentrations were observed on either Day 1 or Day 2. This is in contrast to the findings of Cockcroft and colleagues (8) who reported lower glucose concentrations immediately after high intensity interval cycling during a 3-hour OGTT. Additionally, reduced circulating glucose concentrations were also reported when highintensity cycling was accumulated during the day (22). However, insulin concentrations were lower during Day 1 in ACC; this was particularly pronounced later in the day once three of the four sets of sprints had been accumulated (Figure 2). The lower postprandial insulin concentrations observed are indicative of a reduced insulin requirement to maintain normoglycaemia, but without mechanistic insight it remains unclear if this resulted from changes in insulin sensitivity per se or from other insulin-independent mechanisms. No differences were observed in HOMA-IR or insulin concentrations during Day 2 of the BLO or ACC compared to CON. This is likely related to any effect of exercise in healthy adolescents being short lived, lasting only $24 \mathrm{~h}$ at most (39). The discrepancy between the present Day 1 glucose data and those of previous research are most likely due to the differences in study design and, more specifically, because the current study did not incorporate an oral glucose tolerance test (OGTT). Although the mixed-macronutrient test meals provided a glycaemic challenge reflective of typical dietary practices, the blood sampling timings were scheduled around the natural breaks in the school day and were less frequent than those taken during an OGTT and at times where plasma glucose 
homoeostasis was restored. Thus, the spacing and timing of blood samples likely precluded the detection of potentially subtle changes in glycaemic regulation.

Physiological and affective responses to the maximal sprint exercise differed depending on the pattern of exercise accrual. Average exercise heart rate, indicative of physiological stress, was lower during ACC than BLO despite instructions to perform every sprint as fast as possible. Additionally, participants reported more positive affective ratings after the final two sets of sprint bouts during ACC compared with the corresponding bouts performed during BLO (Table 2). Although these differences were quantitatively small, it is reasonable to suggest that the difference between feeling fairly good vs. fairly bad represents a meaningful shift in affective response and indicates that maximal sprint running was better tolerated when accumulated in short bouts throughout the day. Anecdotally, participants also reported feeing "more tired" and "hotter" during BLO compared with ACC, and often described the former pattern of exercise to be "harder". Importantly, all participants were able to complete all sprint repetitions during both exercise conditions, suggesting that sprint running was better tolerated than previous shortduration, maximal-effort sprint cycling which $33 \%$ of participants were unable to complete due to adverse exercise-induced symptoms (19). These findings are important as it has been suggested that both exercise intensity and affective responses to exercise may be important predictors of long-term exercise adherence (40). Therefore, the finding that ACC was equally efficacious as BLO in reducing [TAG], despite eliciting a lower heart rate and more positive affective perceptions, may have important implications for long-term exercise enjoyment and compliance. However, more comprehensive research examining the psychosocial factors (e.g. enjoyment, self-competence, long-term compliance and preferred pattern of exercise accrual etc.) 
relevant to the performance of such activity is required to shed further light on the potential utility of high-intensity exercise interventions in the school setting.

Although it was not an aim of the current study to examine the suitability or efficacy of maximal sprint exercise for employment with overweight or obese adolescents, it is noteworthy that, of the nineteen participants recruited to the study, two presented overweight $\left(91^{\text {st }}\right.$ and $95^{\text {th }}$ BMI percentile, respectively) and one classified as obese $\left(98^{\text {th }}\right.$ BMI percentile). These participants were able to complete all maximal intensity sprints during both ACC and BLO and reported affective ratings comparable to those of their healthy weight peers (Table 2). After both exercise conditions, all three participants exhibited reduced TAG-TAUC compared with CON (12 to 22\% reduction after ACC; 19 to $32 \%$ reduction after BLO). These data provide preliminary, albeit limited, support for the potential suitability of maximal sprint exercise across a wide spectrum of paediatric populations. Future studies are warranted to further explore the utility of maximal sprint exercise for interventional use with "as risk" children and adolescents.

A number of limitations of the current study should be acknowledged. First, the reliance upon metabolically healthy volunteer participants; all recruited participants were in good general health, and were unlikely to be at risk for chronic disease currently (e.g. obesity, diabetes mellitus, dyslipidaemia). Although the current findings provide valuable "proof of concept", the greatest benefits of this type of in-school intervention would likely be conferred to children and adolescents presenting with the early manifestations of metabolic disorder. Future research is warranted to explore the potential of in-school exercise to target clinical paediatric populations 
and combat metabolic disorder and disease progression. Additionally, the employment of an inschool exercise protocol largely dictated the study design. More frequent blood sampling, in closer proximity to meal consumption, would have been advantageous to investigate subtler glycaemic and insulinaemic responses; however, this represents the reality of translational experimental research and a compromise that was necessary to bridge the gap between the laboratory and free-living settings relevant to young people.

The current study extends the limited body of previous research in a number of ways. First, the translation of high intensity exercise into an ecologically valid setting represents an important advancement. The findings suggest that the time children and adolescents spend at school may be particularly amenable to the accumulation of efficacious high-intensity interval exercise (e.g. maximal sprinting) during the short, regular breaks in the daily timetable. The school setting may, therefore, represent an ideal site for physical activity intervention. This is the first study to demonstrate that sprint running accumulated during the school day reduces postprandial lipaemia and insulinaemia and that this pattern of exercise accrual is more tolerable than the same dose of exercise performed in a single uninterrupted exercise session. The accumulation of high-intensity exercise whilst at school represents a feasible, time-efficient and most importantly efficacious means of promoting metabolic health during youth. Future research is warranted to elucidate both the optimal and minimum number of maximal-intensity sprints, as well as the chronic effects of this form of exercise intervention. 


\section{CONCLUSION}

In conclusion, short-duration sprint running, accumulated in four separate bouts $(<5$ min) during the school day, reduced postprandial triacylglycerol and insulin concentrations in adolescent boys. The exercise-induced reductions observed the day after accumulated exercise were similar in magnitude to those observed after the same volume of exercise was performed in a single block after-school. The current findings demonstrate that in-school, maximal intensity sprint exercise is an efficacious and tolerable alternative to continuous, moderate intensity exercise and HIIE involving longer exercise bouts.

\section{ACKNOWLEDGEMENTS}

This research was supported by the National Institute for Health Research (NIHR) Leicester Biomedical Research Centre. The views expressed are those of the authors and not necessarily those of the NHS, the NIHR or the Department of Health.

\section{CONFLICT OF INTEREST}

The authors declare no conflict of interest. The results of the present study do not constitute endorsement by ACSM. The results of the study are presented clearly, honestly, and without fabrication, falsification, or inappropriate data manipulation. 


\section{REFERENCES}

1) Zilversmit DB. Atherogenesis: a postprandial phenomenon. Circulation. 1979;60:473-85.

2) Nordestgaard BG, Benn M, Schnohr P, Tybjærg-Hansen A. Nonfasting triglycerides and risk of myocardial infarction, ischemic heart disease, and death in men and women. JAMA. 2007;298:299-308.

3) Zeek P. Juvenile atherosclerosis. Arch Pathol Lab Med. 1930;10:417-46.

4) Strong JP, McGill HC. The natural history of coronary atherosclerosis. Am J Pathol. $1962 ; 40: 37-49$.

5) McGill HC, McMahan CA, Herderick EE, Malcom GT, Tracy RE, Strong JP. Origin of atherosclerosis in childhood and adolescence. Am J Clin Nutr. 2000;72:1307S-15S.

6) Lascar N, Brown J, Pattison H, Barnett AH, Bailey CJ, Bellary S. Type 2 diabetes in adolescents and young adults. The Lancet Diabetes \& Endocrinology. 2018;6(1):69-80.

7) Tolfrey K, Thackray AE, Barrett LA. Acute exercise and postprandial lipemia in young people. Pediatr Exerc Sci. 2014;26(2):127-37.

8) Cockcroft EJ, Williams CA, Tomlinson OW, et al. High intensity interval exercise is an effective alternative to moderate intensity exercise for improving glucose tolerance and insulin sensitivity in adolescent boys. J Sci Med Sport. 2015;18(6):720-4.

9) Cooper AR, Goodman A, Page AS, et al. Objectively measured physical activity and sedentary time in youth: The International children's accelerometry database (ICAD). Int. $J$ Behav Nutr Phys Act. 2015;12(1):113.

10) Hallal PC, Andersen LB, Bull FC, Guthold R, Haskell W, Ekelund U. Global physical activity levels: surveillance progress, pitfalls, and prospects. Lancet. 2012;380:247-57. 
11) Gibala MJ. High-intensity interval training: a time-efficient strategy for health promotion? Curr Sports Med Rep. 2007;6(4):211-3.

12) Hebestreit H, Mimura K, Bar-Or O. Recovery of muscle power after high-intensity shortterm exercise: Comparing boys and men. J Appl Physiol. 1993;74(6):2875-80.

13) Baquet G, Stratton G, Van Praagh E, Berthoin S. Improving physical activity assessment in prepubertal children with high-frequency accelerometry monitoring: A methodological issue. Prev Med. 2007;44(2):143-7.

14) Hoos MB, Kuipers H, Gerver WJM, Westerterp KR. Physical activity pattern of children assessed by triaxial accelerometry. Eur J Clin Nutr. 2004;58(10):1425-8.

15) Crisp NA, Fournier PA, Licari MK, Braham R, Guelfi KJ. Adding sprints to continuous exercise at the intensity that maximises fat oxidation: implications for acute energy balance and enjoyment. Metabolism. 2012;61:1280-8.

16) Malik A, Williams C, Bond, B, Weston K, Barker A. Acute cardiorespiratory, perceptual and enjoyment responses to high-intensity interval exercise in adolescents. Eur. J. Sport Sci. 2017;17(10):1335-42.

17) Buchan DS, Ollis S, Young JD, et al. The effects of time and intensity of exercise on novel and established markers of CVD in adolescent youth. Am J Hum Biol. 2011;23(4): 517-26.

18) Tolfrey K, Smallcombe JW. High-intensity interval training. In: Armstrong A, van Mechelen W, editors. Oxford Textbook of Children's Sport and Exercise Medicine. Oxford: Oxford University Press; 2017. p. 477-93.

19) Sedgwick MJ, Morris JG, Nevill ME, Barrett LA. Effect of repeated sprints on postprandial endothelial function and triacylglycerol concentrations in adolescent boys. J Sports Sci. 2015;33:806-16. 
20) Thackray AE, Barrett LA, Tolfrey K. Acute high-intensity interval running reduces postprandial lipemia in boys. Med Sci Sports Exerc. 2013;45:1277-84.

21) Thackray AE, Barrett LA, Tolfrey K. High-intensity Running and Energy Restriction Reduce Postprandial Lipemia in Girls. Med Sci Sports Exerc. 2016;48(3):402-11.

22) Bond B, Williams CA, Jackman SR, Woodward A, Armstrong N, Barker AR. Accumulating exercise and postprandial health in adolescents. Metabolism. 2015;64:1068-76.

23) Chesham RA, Booth JN, Sweeney EL, Ryde GC, Gorely T, Brooks N, Moran C. The Daily Mile makes primary school children more active, less sedentary and improves their fitness and body composition: a quasi-experimental pilot study. BMC Medicine. 2018;16:64.

24) Maraki MI, Sidossis LS. The latest on the effect of prior exercise on postprandial lipaemia. Sports Med. 2013;43:463-81.

25) Slaughter MH, Lohman TG, Boileau RA, et al. Skinfold equations for estimation of body fatness in children and youth. Hum Biol. 1988;60(5):709-23.

26) McCarthy HD, Jarrett KV, Emmett PM, Rogers I. Trends in waist circumferences in young British children: a comparative study. Int J Obesity. 2005;29:157-62.

27) Tanner JM. Growth at adolescence. 2nd ed. Blackwell, Oxford (UK): Blackwell Scientific Publications; 1962. 28-39 p.

28) Robertson RJ, Goss FL, Boer NF, et al. Children's OMNI scale of perceived exertion: mixed gender and race validation. Med Sci Sports Exerc. 2000;32(2):452-8.

29) Poole DC, Wilkerson DP, Jones AM. Validity of criteria for establishing maximal O2 uptake during ramp exercise tests. Eur J Appl Physiol. 2008;102:403.

30) Barker AR, Williams CA, Jones AM, et al. Establishing maximal oxygen uptake in young people during a ramp cycle test to exhaustion. Br J Sports Med. 2011;45:498-503. 
31) Hardy C, Rejeski W. Not What, but How One Feels: The Measurement of Affect during Exercise. J Sport Exerc Psychol. 1989;11(3):304-17.

32) Smallcombe JW, Barrett LA, Morris J, Sherar LB, Tolfrey, K. Small-sided soccer in school reduces postprandial lipaemia in adolescent boys. Med Sci Sports Exerc. 2018;50(11):23519.

33) Matthews DR, Hosker JP, Rudenski AS, Naylor BA, Treacher DF, Turner RC. Homeostasis model assessment: insulin resistance and beta-cell function from fasting plasma glucose and insulin concentrations in man. Diabetologia. 1985;28:412-9.

34) Cohen J. Statistical power analysis for the behavioural sciences. 2nd ed. Hillsdale, New Jersey: Lawrence Erlbaum Associates; 1988.

35) Thackray AE, Barrett LA, Tolfrey K. High-intensity Running and Energy Restriction Reduce Postprandial Lipemia in Girls. Med Sci Sports Exerc. 2016;48(3):402-11.

36) Gill JM, Hardman AE. Exercise and postprandial lipid metabolism: an update on potential mechanisms and interactions with high-carbohydrate diets (review). J Nutr Biochem. 2003;14(3):122-32.

37) Peddie MC, Rehrer NJ, Perry TL. Physical activity and postprandial lipidemia: are energy expenditure and lipoprotein lipase activity the real modulators of the positive effect? Prog Lipid Res. 2012;51:11-22.

38) Seip RL, Semenkovich CF. Skeletal muscle lipoprotein lipase: molecular regulation and physiological effects in relation to exercise. Exerc Sport Sci Rev. 1998;26:191-218.

39) Cockcroft EJ, Williams CA, Weaver H, et al. Acute Exercise and Insulin Sensitivity in Boys: A Time-Course Study. Int J Sports Med. 2017;38(13):967-74.

40) Ekkekakis P, Parfitt G, Petruzzello S. The Pleasure and Displeasure People Feel When They Exercise at Different Intensities. Sports Medicine. 2011;41(8):641-67. 


\section{LIST OF FIGURES}

Figure 1a Study protocol for Day 1 and the 24 hours preceding (Day 0). Test meals were standardised. TAG - triacylglycerol. ACC and BLO were completed separately in a within measures cross-over design. During the standard-practice control condition (CON) no exercise was performed, however, test meals and blood samples were as depicted above. Capillary blood samples were taken during natural breaks in the school day.

Figure 1b Study protocol for Day 2. Test meals were standardised to body mass. TAG triacylglycerol

Figure 2 Fasting (0) and postprandial TAG, insulin and glucose concentrations during the three experimental conditions on Day 1 and Day 2. Black rectangles represent the consumption of breakfast and lunch, respectively. $*$ denotes significant difference between ACC and CON TAUC $(\mathrm{P}<0.05)$. \# denotes significant difference between BLO and CON TAUC $(\mathrm{P}<0.05)$. 
Figure 1a

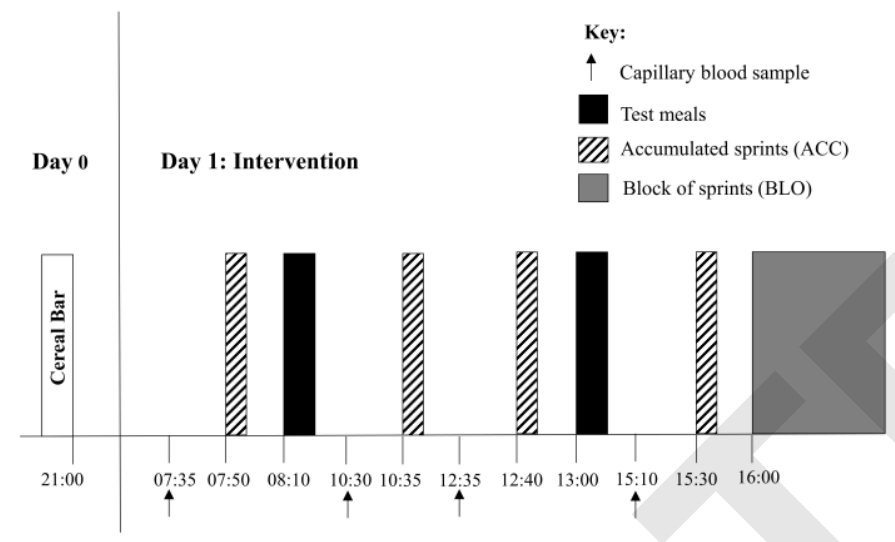


Figure 1b

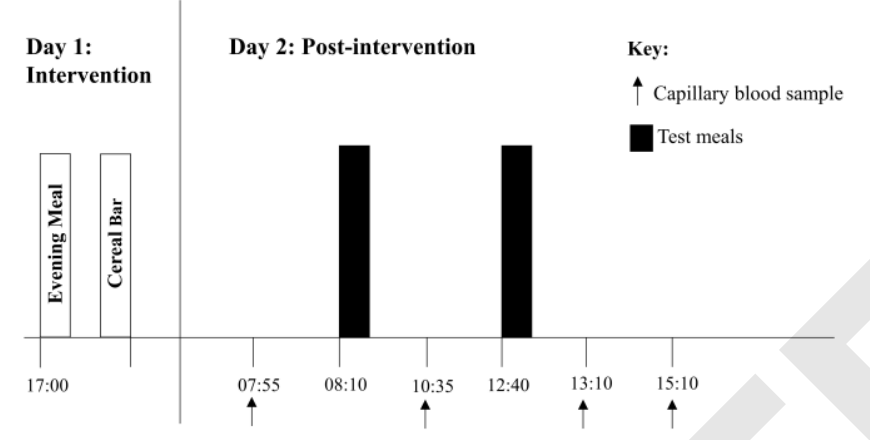


Figure 2

[TAG] Day 1

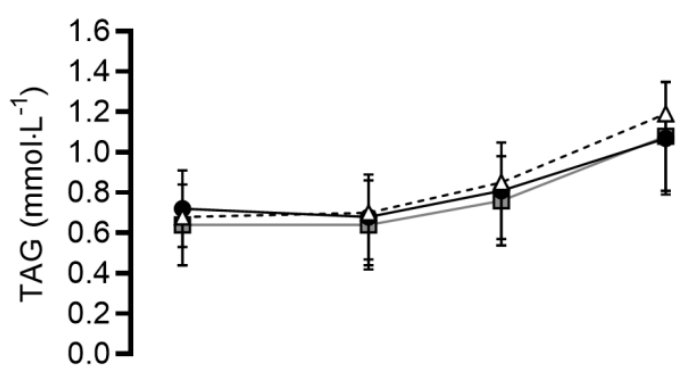

[Glucose] Day 1

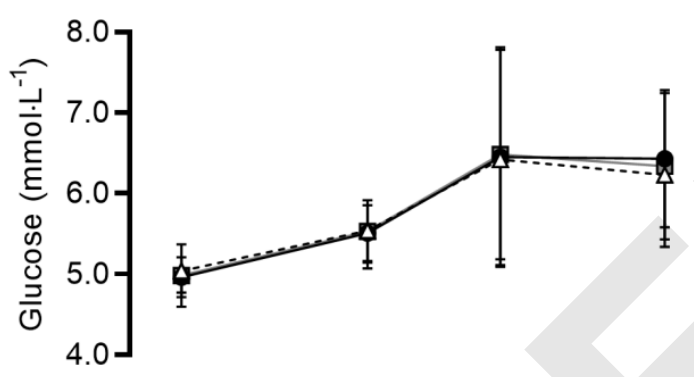

[Insulin] Day 1

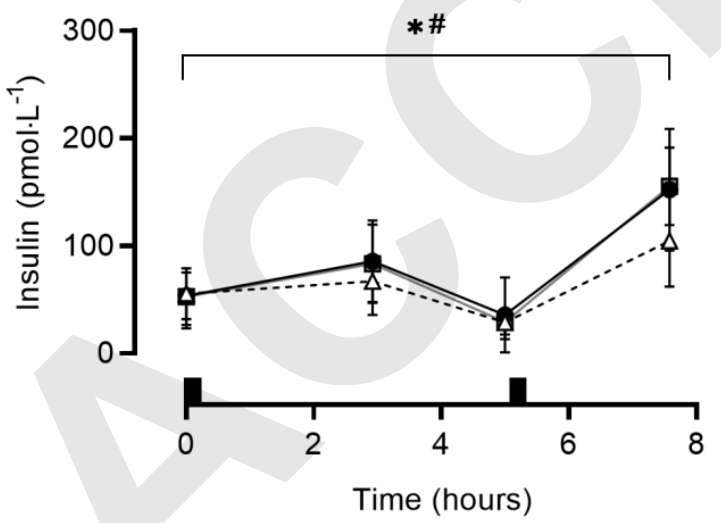

[TAG] Day 2

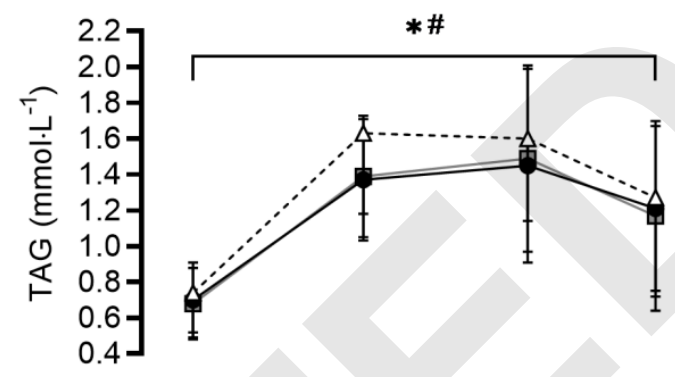

[Glucose] Day 2

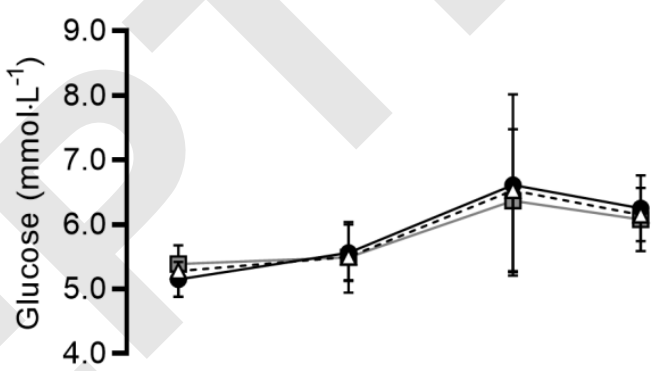

[Insulin] Day 2

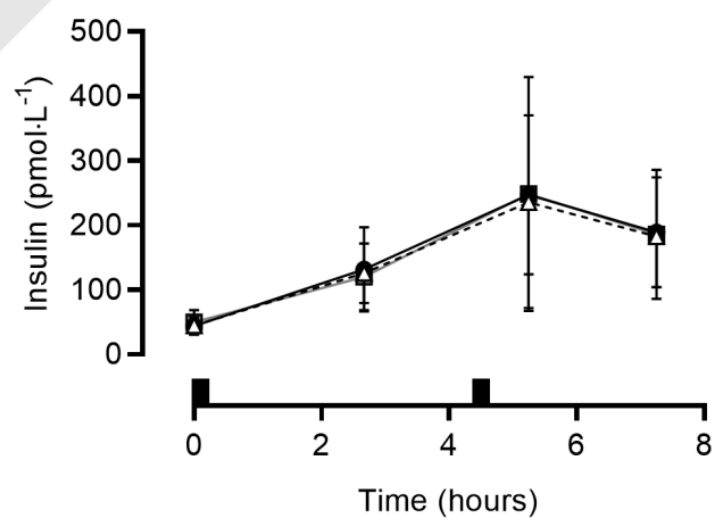

$\because-\cdot$ ACC $\bullet$ BLO $\square$ CON 
Table 1 Participant characteristics $(n=19)$

\begin{tabular}{lcccc}
\hline & Mean (SD) & \multicolumn{2}{c}{ Range } \\
\hline Age $(\mathrm{y})$ & $12.3(0.7)$ & 11.2 to 13.3 \\
Body mass $(\mathrm{kg})$ & $43.1(9.0)$ & 29.2 to 62.6 \\
Stature $(\mathrm{m})$ & $1.56(0.10)$ & 1.42 to 1.74 \\
Body mass index $\left(\mathrm{kg} \cdot \mathrm{m}^{-2}\right)$ & $17.6(2.8)$ & 12.5 to 24.7 \\
Waist circumference $(\mathrm{cm})$ & $55.1(17.9)$ & 22.4 to 81.5 \\
Genital development* & $3(1)$ & 1 to 3 \\
Pubic hair development* & $3(1)$ & 1 to 3 \\
Body fat $(\%)$ & $15.3(5.2)$ & 8.7 to 31.3 \\
Peak $\dot{\mathrm{V}} \mathrm{O}_{2}\left(\mathrm{~mL} \cdot \mathrm{kg}^{-1} \cdot \mathrm{min}^{-1}\right)$ & $53.3(6.1)$ & 37.8 to 62.0 \\
\hline
\end{tabular}

* 1 to 5 stages (median (interquartile range)) 
Table 2 Heart rate and affective responses across sets of maximal sprints during ACC and BLO

\begin{tabular}{|c|c|c|c|c|}
\hline & \multicolumn{2}{|c|}{ Heart Rate (bpm) } & \multicolumn{2}{|c|}{ One Item Feeling Scale $(-5$ to +5$)$} \\
\hline & ACC (SD) & $\mathrm{BLO}(\mathrm{SD})$ & $\mathrm{ACC}(\mathrm{SD})$ & $\mathrm{BLO}(\mathrm{SD})$ \\
\hline Set 1 & $177(9)$ & $178(10)$ & +1 (2) [Fairly Good $]$ & +1 (1) [Fairly Good $]$ \\
\hline Set 2 & $176(8)$ & $184(10)^{*}$ & +1 (1) $[$ Fairly Good $]$ & +1 (1) [Fairly Good $]$ \\
\hline Set 3 & $175(8)$ & $182(11)^{*}$ & +1 (1) [Fairly Good $]$ & $0(2)[\text { Neutral }]^{*}$ \\
\hline Set 4 & $175(8)$ & $182(11)^{*}$ & +1 (2) $[$ Fairly Good $]$ & $-1(1)[\text { Fairly bad }]^{*}$ \\
\hline
\end{tabular}

* Denotes significant difference between ACC and BLO $(P<0.005)$ 
Table 3 Total area under the curve (TAUC) for postprandial [TAG], [insulin] and [glucose] on Day 1 and Day 2

\section{Ratio Difference \% $(95 \% \mathrm{CI})$}

\begin{tabular}{|c|c|c|c|c|c|c|}
\hline & $\mathrm{CON}$ & $\mathrm{ACC}$ & BLO & ACC vs. CON & BLO vs. CON & BLO vs. ACC \\
\hline $\begin{array}{l}\text { Day } 1 \text { TAUC-TAG } \\
\left(\mathrm{mmol} \cdot \mathrm{L}^{-1}\right)\end{array}$ & $0.71(0.62$ to 0.82$)$ & $0.79(0.69$ to 0.90$)$ & $0.77(0.67$ to 0.88$)$ & $11(-4$ to 28$)$ & $8(-7$ to 24$)$ & $-3(-16$ to 12$)$ \\
\hline $\begin{array}{l}\text { Day } 1 \text { TAUC-insulin } \\
\left(\mathrm{pmol} \cdot \mathrm{L}^{-1}\right)\end{array}$ & $66.4(56.0$ to 78.9$)$ & $51.8(43.6$ to 61.4$)$ & 69.7 (58.8 to 82.8$)$ & $-22(-32 \text { to }-10)^{*}$ & $5(-9$ to 21$)$ & $35(17 \text { to } 56)^{*}$ \\
\hline $\begin{array}{l}\text { Day } 1 \text { TAUC- } \\
\text { glucose }\left(\mathrm{mmol} \cdot \mathrm{L}^{-1}\right)\end{array}$ & $6.76(6.44$ to 7.10$)$ & $6.71(6.39$ to 7.06$)$ & $6.73(6.41$ to 7.08$)$ & $-1(-3$ to 2$)$ & $0(-2$ to 2$)$ & $0(-2$ to 3$)$ \\
\hline $\begin{array}{l}\text { Day } 2 \text { TAUC-TAG } \\
\left(\mathrm{mmol} \cdot \mathrm{L}^{-1}\right)\end{array}$ & 1.35 (1.19 to 1.55$)$ & $1.19(1.04$ to 1.36$)$ & $.23(1.07$ to 1.40$)$ & $-12(-19 \text { to }-5)^{*}$ & $-10(-17 \text { to }-2)^{*}$ & $3(-39$ to 12$)$ \\
\hline $\begin{array}{l}\text { Day } 2 \text { TAUC-insulin } \\
\left(\mathrm{mmol} \cdot \mathrm{L}^{-1}\right)\end{array}$ & $141.9(116.6$ to 172.7$)$ & 139.9 (115.3 to 169.8$)$ & $150.8(124.4$ to 182.7$)$ & $-1(-19$ to 20$)$ & $-6(-13$ to 29$)$ & $8(-11$ to 31$)$ \\
\hline $\begin{array}{l}\text { Day } 2 \text { TAUC-glucose } \\
\left(\mathrm{mmol} \cdot \mathrm{L}^{-1}\right)\end{array}$ & $6.79(6.52$ to 7.07$)$ & $6.82(6.56$ to 7.10$)$ & $6.73(6.47$ to 7.00$)$ & $1(-3$ to 5$)$ & $1(-2$ to 5$)$ & $1(-3$ to 4$)$ \\
\hline
\end{tabular}

CON - standard-practice control (no sprints); ACC - accumulated in-school sprint running; BLO - a single block of afterschool sprint running

Values are geometric means and corresponding $95 \%$ CI for $n=19$. Pairwise comparisons are percentage difference $(\%)$ based on ratios of geometric means and corresponding 95\% CI (\%). Statistical analyses are based on natural log transformed data. * Statistically significant difference $(\mathrm{P}<0.05)$. 